Supporting Information

\title{
Interlayer design of pillared graphite by Na-halide cluster intercalation for anode material of sodium-ion battery
}

Taesoon Hwang ${ }^{\dagger}$, Maenghyo $\mathrm{Cho}^{\dagger *}$ and Kyeongjae $\mathrm{Cho}^{\ddagger *}$

†Department of Mechanical and Aerospace Engineering, Seoul National University, 1 Gwanak-ro, Gwanak-gu, Seoul 08826, Republic of Korea.

‡ Department of Materials Science and Engineering, University of Texas at Dallas, Richardson, TX 75080, USA.

\section{Corresponding Author}

*(Maenghyo Cho); e-mail:mhcho@snu.ac.kr.

*(Kyeongjae Cho); e-mail: kjcho@utdallas.edu. 

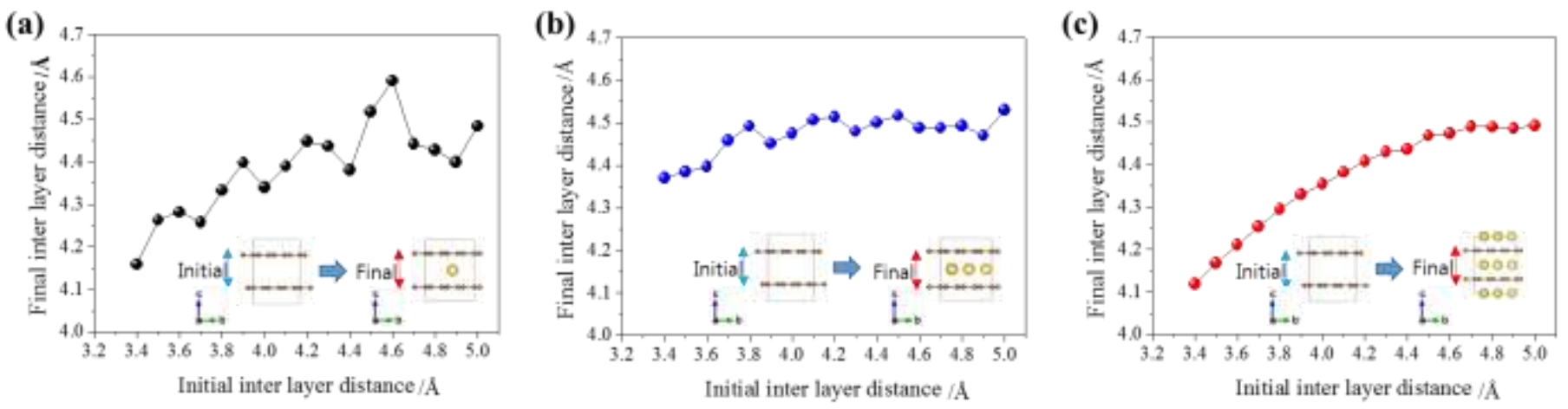

Figure S1. Relaxed interlayer of sodium-ion intercalation into expanded graphite as variations of initially pre expanded interspacing for $1 \mathrm{~mol}$ (a), $2 \mathrm{~mol}$ (b) and $3 \mathrm{~mol}$ (c) of sodium-ions. 


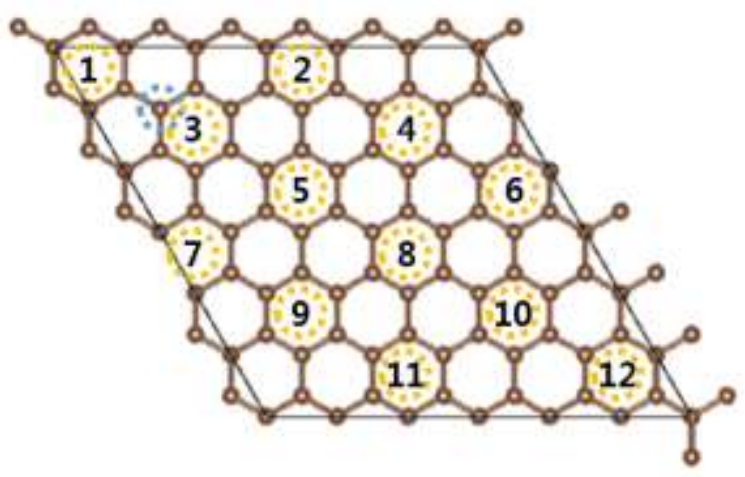

Figure S2. sodium-ion and halogen ion positions in halide ion pre intercalated GICs of $6 \times 6 \times 6$ unit cell. (blue dashed circle : halide ion position and yellow dashed circle : sodium-ion position) 
(a)

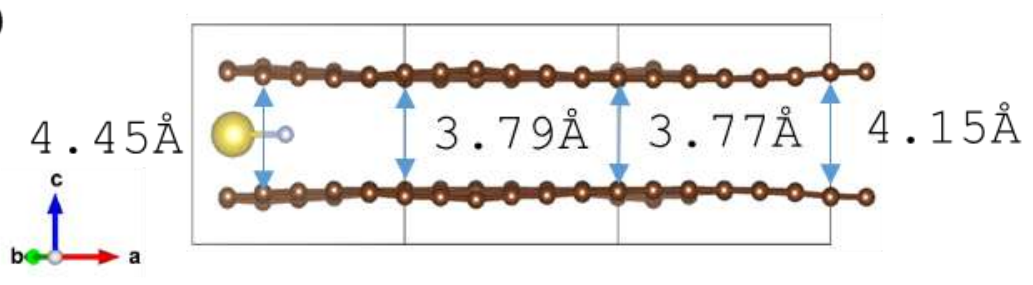

(b)

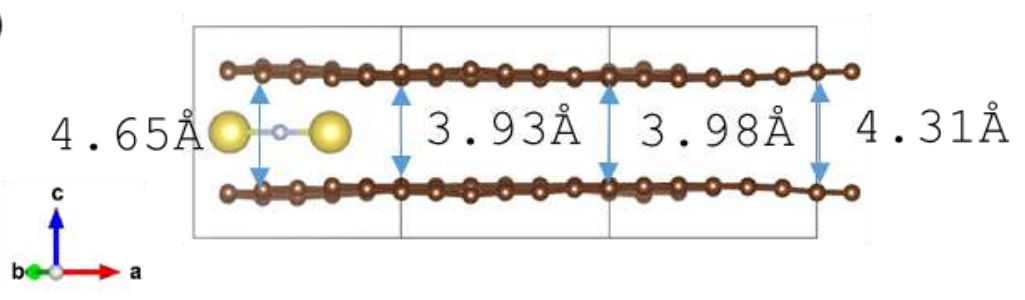

(c)

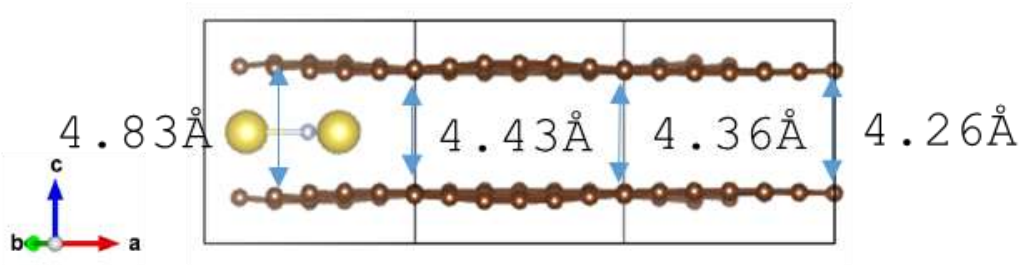

(d)

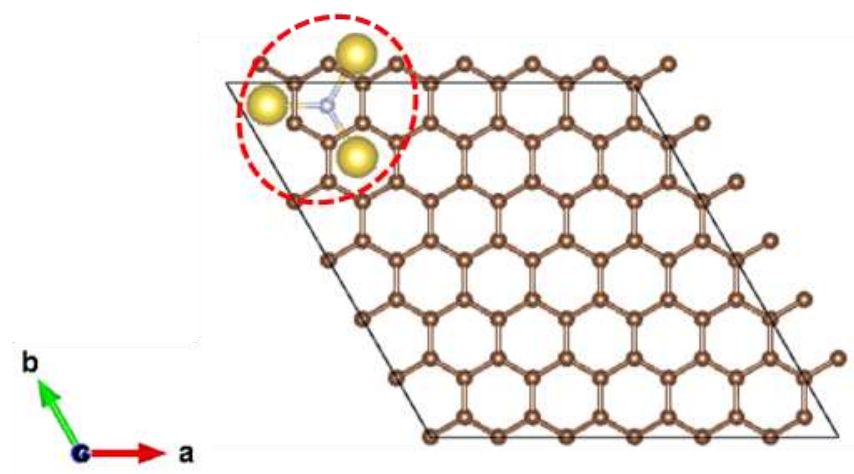

Figure S3. Atomic structure of F ion pre intercalated GICs of $6 \times 6 \times 6$ unit cell for $1 \mathrm{~mol}$ (a), $2 \mathrm{~mol}$ (b) and 3 mol (c,d) sodium-ion contents(red dashed circle : $\mathrm{Na}_{3} \mathrm{~F}$ cluster). 
(a)

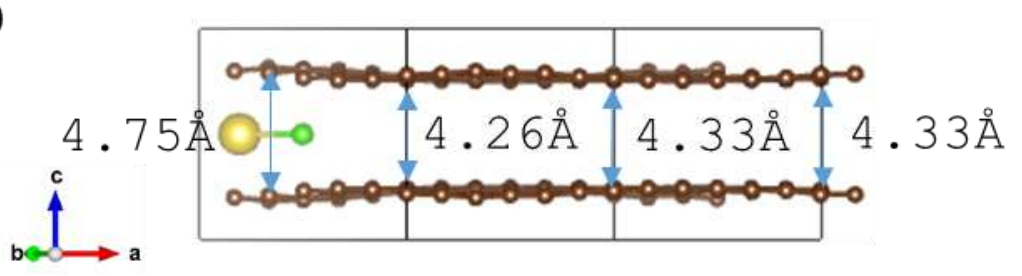

(b)

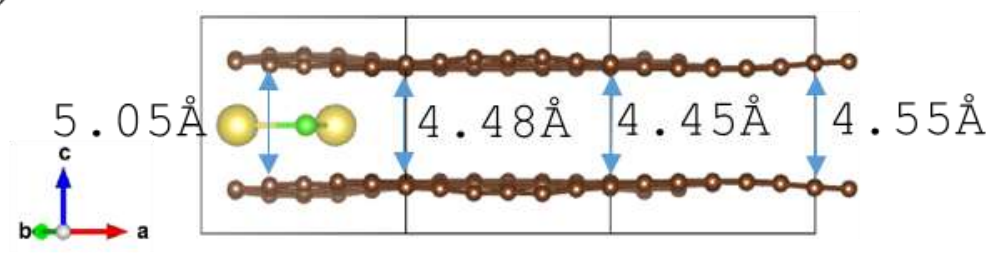

(c)

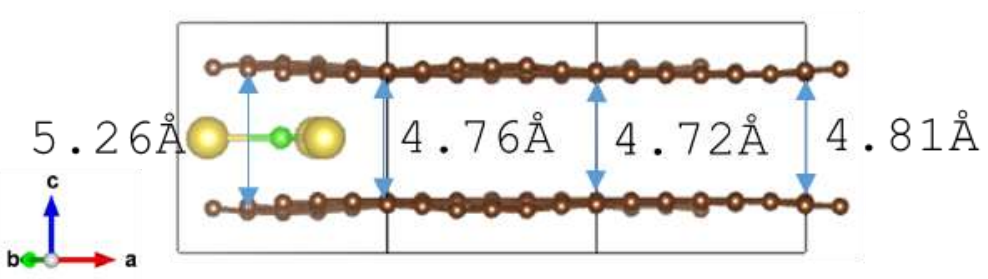

(d)

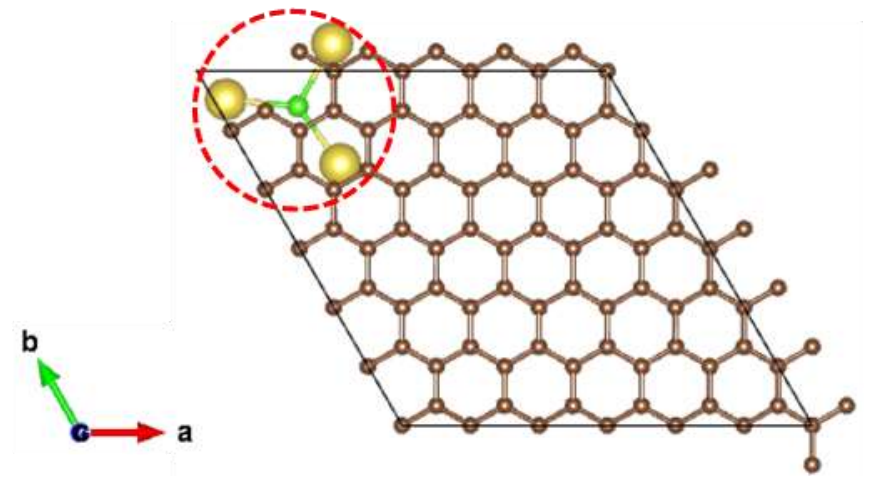

Figure S4. Atomic structure of $\mathrm{Cl}$ ion pre intercalated GICs of $6 \times 6 \times 6$ unit cell for $1 \mathrm{~mol}$ (a), $2 \mathrm{~mol}$ (b) and 3 mol (c,d) sodium-ion contents(red dashed circle : $\mathrm{Na}_{3} \mathrm{Cl}$ cluster). 
(a)

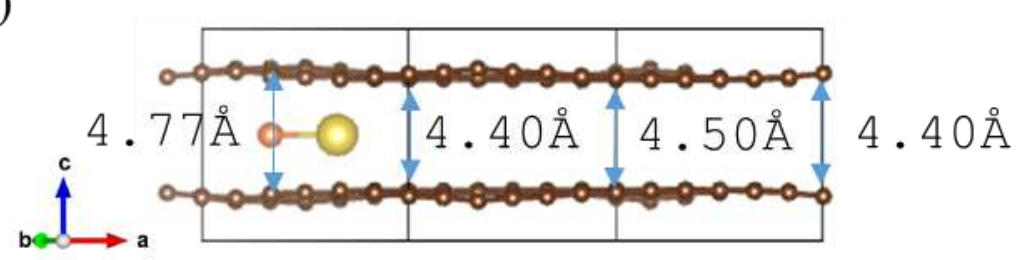

(b)

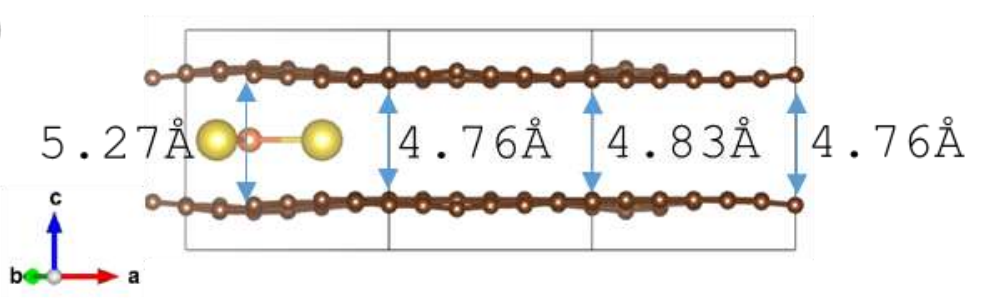

(c)

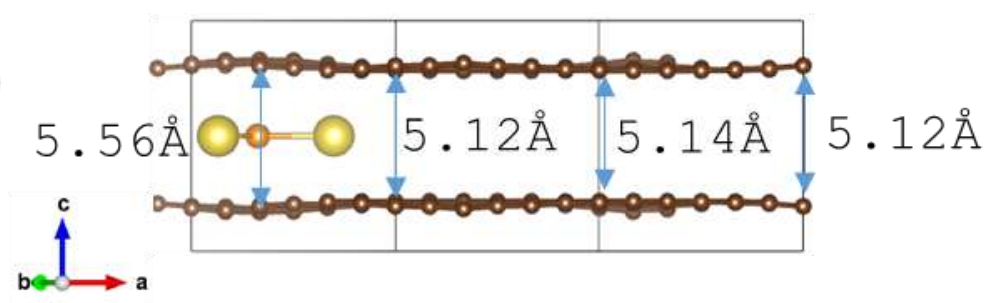

(d)

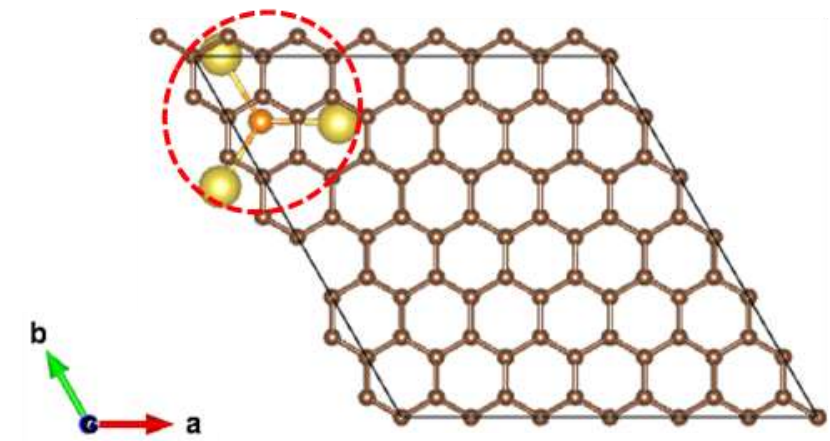

Figure S5. Atomic structure of $\mathrm{Br}$ ion pre intercalated GICs of $6 \times 6 \times 6$ unit cell for $1 \mathrm{~mol}$ (a), $2 \mathrm{~mol}$ (b) and 3 mol (c,d) sodium-ion contents(red dashed circle : $\mathrm{Na}_{3} \mathrm{Br}$ cluster). 
(a)
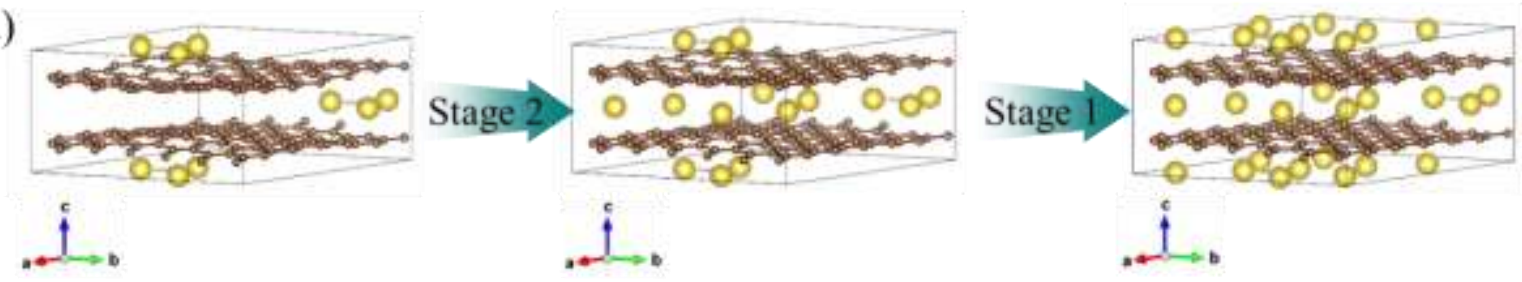

(b)
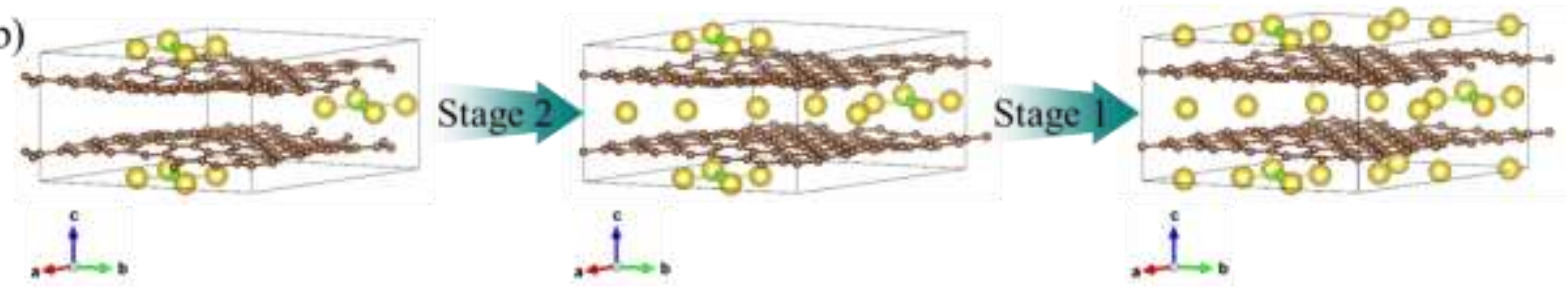

(c)

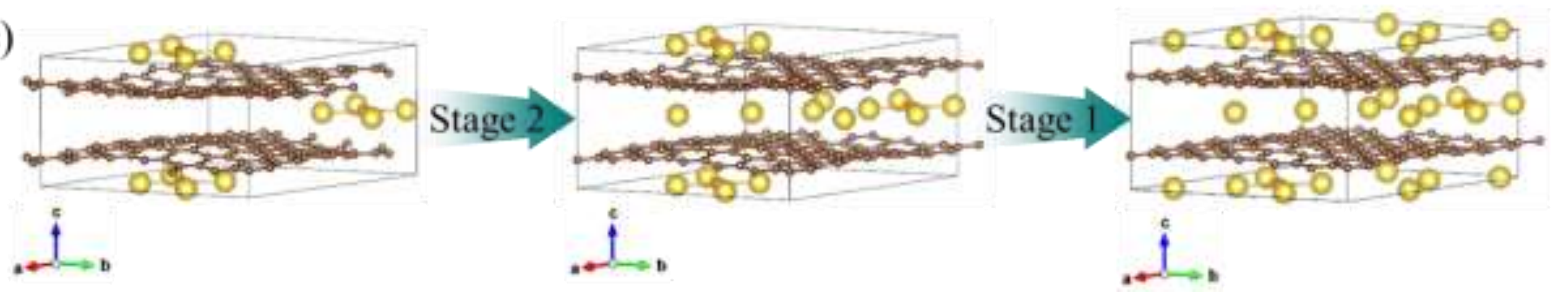

Figure S6. Atomic structures of sodium-ion intercalation into $\mathrm{Na}_{3} \mathrm{~F}$ (a), $\mathrm{Na}_{4} \mathrm{Cl}$ (b) and $\mathrm{Na}_{4} \mathrm{Br}$ (c) cluster GICs from stage 2 to stage 1 . 
Table S1. Formation energies as the variations of sodium-ion position in halogen elements (F, $\mathrm{Cl}$ and $\mathrm{Br})$ pre intercalated GICs (1,3,6 and 11 positions are locations near halogen elements.)

\begin{tabular}{cccc} 
& \multicolumn{3}{c}{ Formation energy / eV } \\
\cline { 2 - 4 } Na ion position & $\mathrm{F}$ & $\mathrm{Cl}$ & $\mathrm{Br}$ \\
\hline 1 & -1.31 & -1.67 & -1.76 \\
2 & -0.49 & -1.14 & -1.37 \\
3 & -1.3 & -1.67 & -1.76 \\
4 & -0.43 & -1.06 & -1.27 \\
5 & -0.43 & -1.08 & -1.32 \\
6 & -1.3 & -1.52 & -1.63 \\
7 & -0.49 & -1.14 & -1.37 \\
8 & -0.37 & -1.02 & -1.25 \\
9 & -0.43 & -1.06 & -1.27 \\
10 & -0.4 & -1.03 & -1.26 \\
11 & -1.3 & -1.52 & -1.63 \\
12 & -0.39 & -1.02 & -1.26
\end{tabular}


Table S2. Average net charges according to sodium-ion contents $(n)$ in $\mathrm{Na}_{n} \mathrm{~F}$ cluster GICs.

\begin{tabular}{cccc}
$\mathrm{n}$ & $\mathrm{F}$ & $\mathrm{Na}_{\mathrm{c}}$ & $\mathrm{Na}_{\mathrm{i}}$ \\
\hline 0 & -0.62 & & \\
1 & -0.74 & 0.87 & \\
2 & -0.85 & 0.88 & \\
3 & -0.88 & 0.89 & \\
4 & -0.88 & 0.89 & 0.88
\end{tabular}

Table S3. Average net charges according to sodium-ion contents $(n)$ in $\mathrm{Na}_{n} \mathrm{Cl}$ cluster GICs.

\begin{tabular}{cccc}
$\mathrm{n}$ & $\mathrm{Cl}$ & $\mathrm{Na}_{\mathrm{c}}$ & $\mathrm{Na}_{\mathrm{i}}$ \\
\hline 0 & -0.34 & & \\
1 & -0.54 & 0.88 & \\
2 & -0.75 & 0.88 & \\
3 & -0.83 & 0.89 & \\
4 & -0.83 & 0.90 & 0.90
\end{tabular}


Table S4. Average net charges according to sodium-ion contents $(n)$ in $\mathrm{Na}_{n} \mathrm{Br}$ cluster GICs.

\begin{tabular}{cccc}
$n$ & $\mathrm{Br}$ & $\mathrm{Na}_{\mathrm{c}}$ & $\mathrm{Na}_{\mathrm{i}}$ \\
\hline 0 & -0.09 & & \\
1 & -0.33 & 0.88 & \\
2 & -0.69 & 0.89 & \\
3 & -0.79 & 0.89 & \\
4 & -0.79 & 0.89 & 0.90
\end{tabular}

Table S5. Average bond length between halogen and $\mathrm{Na} / \AA$.

\begin{tabular}{cccc}
$n$ & $\mathrm{~F}$ & $\mathrm{Cl}$ & $\mathrm{Br}$ \\
\hline 1 & 2.11 & 2.60 & 2.76 \\
2 & 2.08 & 2.58 & 2.75 \\
3 & 2.16 & 2.60 & 2.76 \\
4 & 2.16 & 2.60 & 2.77
\end{tabular}

